\title{
CREATION OF LARGE CATALOGUES BY USING OF VIRTUAL OBSERVATORIES
}

\author{
Yu.I.Protsyuk, O.M.Kovalchuk \\ Research Insitute "Nikolaev Astronomical Observatory", \\ Mykolaiv, Ukraine,yuri@nao.nikolaev.ua
}

\begin{abstract}
We developed an application program to search images in the registers and databases of Virtual Observatories and to download them to local computer. The program has the ability to process XML file in VO Table format to generate links to images, as well as to work directly with the astronomical servers. To improve the efficiency for downloading of large number of images, we used multi-threaded mode. The program runs under the Windows operating system. Using the program in 2014 year, we found and downloaded more than 145 thousand of images of open clusters, having total volume of about 300 GB. Total download time was about 7 days. To process the downloaded images, we created and configured a complex of 10 virtual machines on two PCs for parallel image processing by using Astrometrica program. Total processing time was about 14 days. An application program was also created to analyse the obtained results, which were used to create four catalogues of stellar coordinates at the average epoch of 1953 to 1998 . The total number of stars in the catalogues is more than 35 million. The standard error is 0.04 " to 0.07 ", and the average number of observations is 4 to 6 . The catalogs are used to improve proper motions of stars in and around of open clusters.
\end{abstract}

Keywords: Astrometry - Astronomical data bases Catalogs - Virtual observatory tools.

\section{Introduction}

In recent years through the development of panoramic receivers and information technology astronomers must respond to an avalanche increasing amount of data that contain information on many celestial objects at different wavelengths. Existing and future huge databases can not afford to use in one isolated institution, but provides such opportunities for international cooperation and organization wide access to collected data. International Virtual Observatories Alliance (IVOA) created to pool resources astronomical data at the national and international levels and provide convenient unified search engine to access the data. Since 2011 Ukrainian Virtual Observatory (UkrVO) included to IVOA (Vavilova et al., 2010, 2011, 2012).

New scientific results can be obtained using data accumulated on different instruments for a long time. One example of this is the study of open clusters (OC) of our Galaxy, formations of stars that have a common origin. It helps solve a wide class of problems concerning formation of stars, structure and evolution of the Galaxy and others. Currently, the number of OC estimated near one hundred thousand and only a small percentage of them have been studied in detail. In particular, we don't know the exact distances and middle proper motions of about half of all known OC.

To solve this problem at the Research Institute: Nikolaev Astronomical Observatory (RI NAO) we studied of stars in regions around the OC several last years using the achievements of a decade of work on the creation and implementation of modern information technologies in astronomy, including the UkrVO creation (Protsyuk et al., 2007; Mazhaev et al., 2014; Vavilova I. et al.: 2010, 2011, $2012,2014)$. With the assistance of our own observations and data from IVOA registries received at different times and at different telescopes, an accurate catalogs of positions and proper motions of stars around $\mathrm{OC}$ in creating (Protsyuk et al., 2014).

\section{Develop an application program to search images in VO databases}

Search and downloading of astronomical images obtained at different times and on different instruments through specialized astronomical websites or through existing specialized software requires a big amount of manual work and time if there is a need for a large number of observations. For this purpose we developed methodics and software to search for images in the IVOA registers and astronomical databases (DB) and automatically download them to computer. Software provides features for finding information about astronomical images and automatically download them to your computer when matching predetermined criteria. Search criteria, given in a prepared list, include the angular coordinates and search radius. The program has the ability to process XML files in VOTable format to create links to the images and work directly with astro-servers. Software running in the Windows operating system and consists of several functional modules - namely: content downloads module, module of content analysis of Web page and formation of links to files and image downloads module. The program works in multithreaded mode and provides full control of downloading and processing of possible network errors. Download speed is limited only by the speed of the Internet connection. TThe modular construction principle of the program provides high stability that is essential for automatic downloading large file sets. 


\section{Processing and analysis of the obtained images}

We used developed program for 500 selected fields aropund OC and were found and downloaded more than 145 thousand images in 2014. Images were obtained at various of telescopes from 1952 to 1999 with total capacity near 300GB. Total duration of the work of program was about 7 days of continuous operation. By automating the process productivity increased about 200 times.

For processing the images arrays we created complex of 10 virtual machines on $2 \mathrm{PCs}$, which is configured for parallel automatic processing of large volumes of observations using Astrometrica (http://www.astrometrica.at) with additional program for automation. Images were downloaded from Aladin Images Server (http://aladin.u-strasbg.fr) for infrared photometric reviews 2MASS (Skrutskie M.F. et al., 2006.) and DENIS (DENIS Consortium, 2005) and from the server http://archive.stsci.edu for The Digitized Sky Surveys (DSS) array. Also we include near the 20 thousand own observations at the telescope Mobitel KT-50. The UCAC4 catalog (Zacharias et al., 2013) were used for all reduction as reference one. The total number of processed images - more than 160 thousand. Were received coordinates for more than 235 million objects with good precision. For processing of the entire array of images with 10 virtual machines took about 14 full days job.

Also software for the analysis of the results were created. The accuracy analysis of (O-C) (UCAC4 reference catalog) depending on the Mag, Dec, Ra and Color band. Calculation of the standard deviation and the mean $(\mathrm{O}-\mathrm{C})$ made in 3-iteration. This number of iterations is selected because the number of exceptions by the criterion $3 \sigma$ in third iteration decreases sharply. Part of the results of calculations for one source (2MASS) set out in the form of a graphs of Figures 1-4.

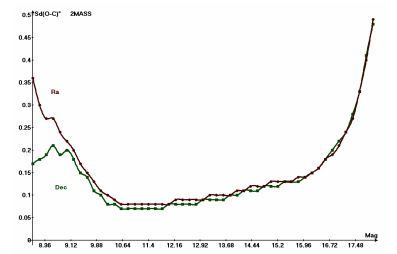

Figure 1: Dependence of the standard deviation (SD) of O-C to magnitude
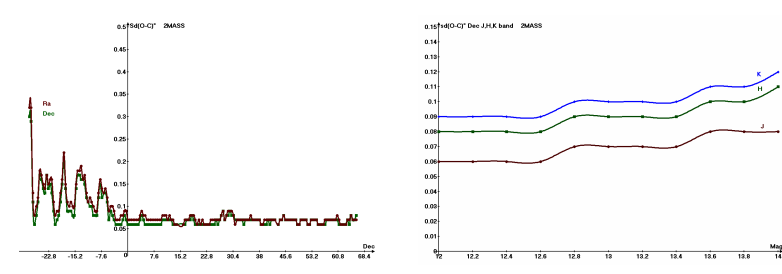

Figure 3: Dependence of the $\mathrm{SD}$ of $\mathrm{O}-\mathrm{C}$ to declination
We made analysis of accuracy of the results from all 4 sources by magnitude, coordinates and color band.

\section{Creation and analysis of the catalogs}

Image processing conducted using the program Astrometrica and additional program, created in the RI $\mathrm{NAO}$, for batch mode processing. Were processed over 160 thousand images with UCAC4 reference catalog. All data is divided into five parts and we obtained five catalogs at different epochs.

First consider the catalog, obtained from 2MASS set. We received more than 135 million objects on it in sites containing OC. With this data we obtained astrometric catalog of over 19.7 million stars $(9-18.5)^{\mathrm{m}}$ (Fig.5) which observed three and more times with the average number of observations (ANO) of a star about 6.3 times and the average accuracy of catalog position (AACP) in right ascension (RA) 40 mas and declination (DEC) 39 mas (Fig. 6). The average epoch of catalog is 1998.7.

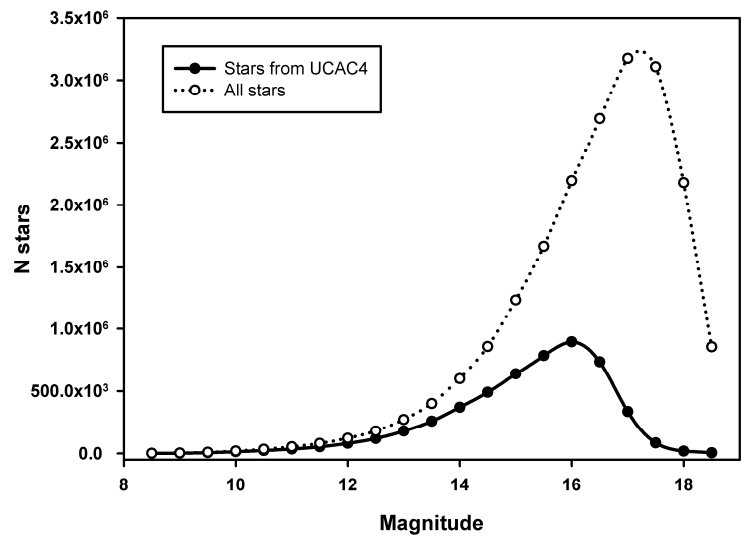

Figure 5: Distribution of stars of 2MASS set catalog to magnitude
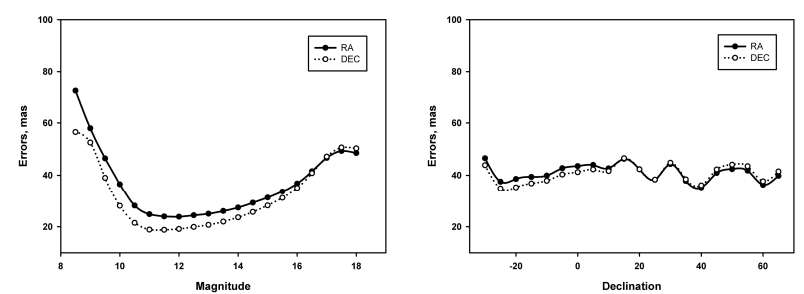

Figure 6: Dependence of precision of 2MASS set catalog to magnitude.

Figure 7: Dependence accuracy of 2MASS set catalog to DEC
As shown in Figure 7 the accuracy distribution to declination without major deviations. In comparison, the reference catalog on the same data, which includes over 5.1 million stars from UCAC4 that were observed 3 or more times with ANO of a one star about 4.9 times have AACP 32 mas by RA and 28 mas by DEC.

Next consider the catalog, obtained from the DENIS set. We received more than 24.5 million objects in regions, containing OC. With this data we obtained astro- 
metric catalog of more than 3 million stars $(9-18.5)^{\mathrm{m}}$ (Fig. 8) which observed three and more times with the ANO of a star about 5.2 times and AACP in RA 52 mas and DEC 68 mas (Fig. 9). The average epoch of catalog is 1998.8

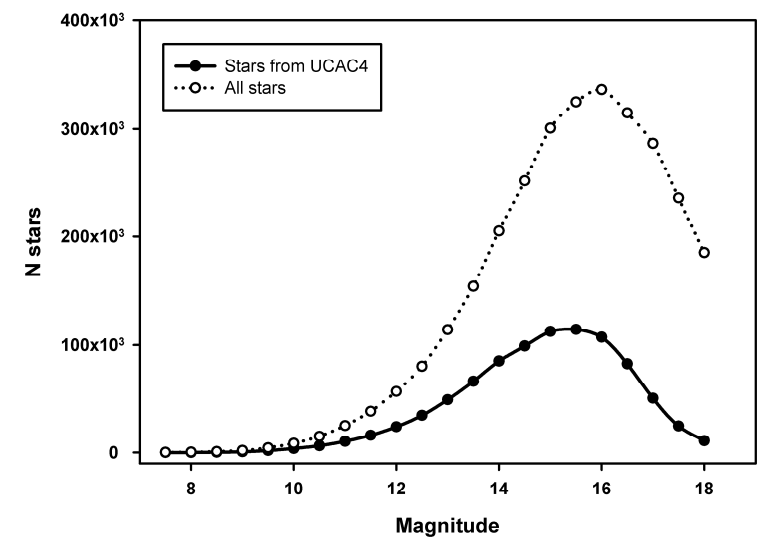

Figure 8: Distribution of stars of DENIS set catalog to magnitude
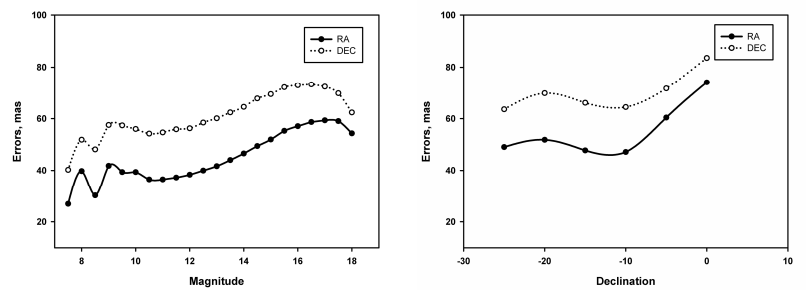

Figure 9: Dependence of precision of DENIS set catalog to magnitude.

Figure 10: Dependence accuracy of DENIS set catalog to DEC

As shown in Figure 10 the accuracy distribution to declination have the noticeable dependence. In comparison, the reference catalog on the same data, which includes over 0.9 million stars from UCAC4 that were observed 3 or more times with ANO of a one star about 5.3 times have AACP 41 mas by RA and 52 mas by DEC.

Next consider the catalog, obtained from the DSS-A set. We received more than 19.5 million objects in regions, containing OC. With this data we obtained astrometric catalog of about 3 million stars (13-18.5) ${ }^{\mathrm{m}}$ (Fig. 11) which observed three and more times with the ANO of a star about 4.1 times and AACP in RA 65 mas and DEC 70 mas (Fig. 12). The average epoch of catalog is 1953.2.

As shown in Figure 13 the accuracy distribution to declination have the noticeable dependence with accuracy deviation. In comparison, the reference catalog on the same data, which includes about 0.7 million stars from UCAC4 that were observed 3 or more times with ANO of a one star about 3.6 times have AACP 65 mas by RA and 70 mas by DEC.

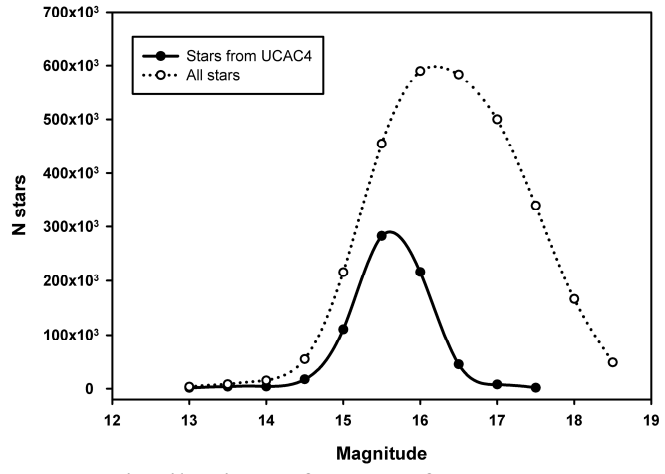

Figure 11: Distribution of stars of DSS-A set catalog to magnitude

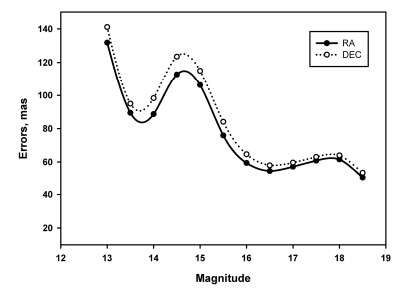

Figure 12: Dependence of precision of DSS-A set catalog to magnitude.
Figure 13: Dependence accuracy of DSS-A set catalog to DEC

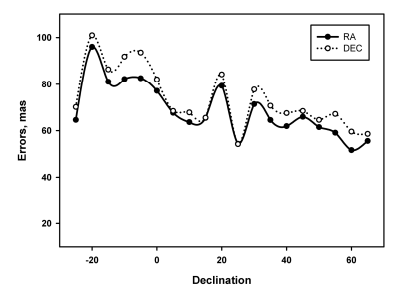

Next consider the catalog, obtained from the DSS-B set. We received more than 52.5 million objects in regions, containing OC. With this data we obtained astrometric catalog of more than 8 million stars (13-18.5) ${ }^{\mathrm{m}}$ (Fig. 14) which observed three and more times with the ANO of a star about 5.7 times and AACP in RA 47 mas and DEC 54 mas (Fig.15). The average epoch of catalog is 1988.3 .

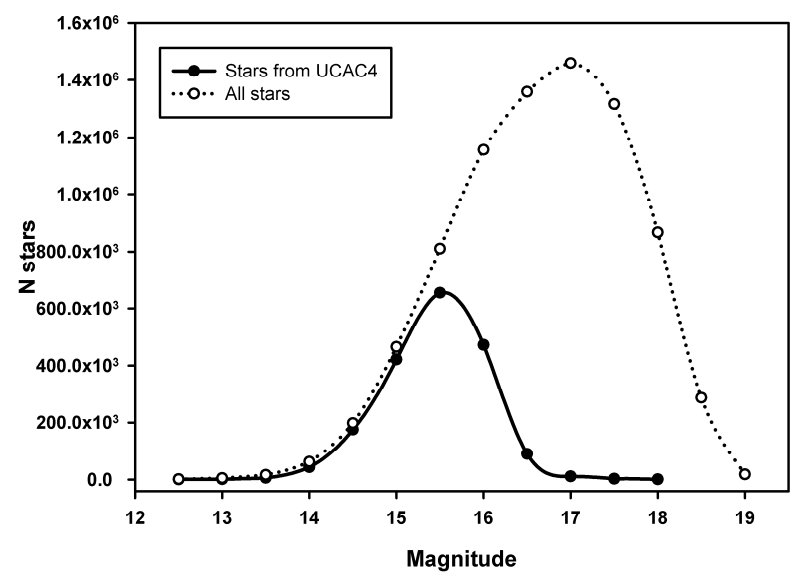

Figure 14: Distribution of stars of DSS-B set catalog to magnitude 

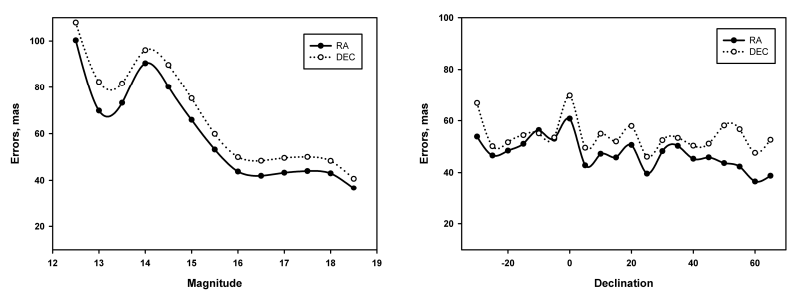

Figure 15: Dependence of precision of DSS-B set catalog to magnitude. accuracy of DSS-B set catalog to DEC
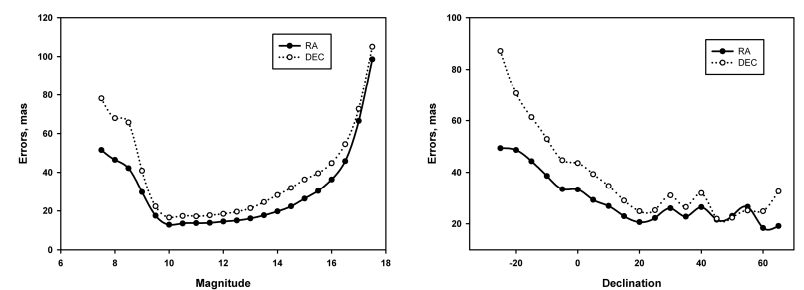

Figure 18: Dependence of precision of MOBITEL set catalog to magnitude.
As shown in Figure 16 the accuracy distribution to declination have the some dependence and the picture is somewhat reminiscent of the behavior of the system in the Mobitel telescope (Fig. 19) but zero-point is not at zenith but around the equator. When you move to north accuracy difference between the two coordinates increases. In comparison, the reference catalog on the same data, which includes over 1.8 million stars from UCAC4 that were observed 3 or more times with ANO of a one star about 5.6 times have AACP 50 mas by RA and 56 mas by DEC. This is worse, than accuracy of general catalog and is associated with significantly worse accuracy of bright stars.

Finally consider the MOBITEL-2014 catalog, which include data from more than 20 thausands of CCD images, observed in RI NAO in 2011-2014. We received more than 90 million objects in regions, containing OC in the area of \pm $20^{\circ}$ from the Galactic plane. With this data we obtained astrometric catalog of over 3.7 million stars $(8-17)^{\mathrm{m}}$ (Fig. 17) which observed three and more times with the ANO of a star about 21 times and AACP in RA 29 mas and DEC 38 mas (Fig. 18). The average epoch of catalog is 2013.6.

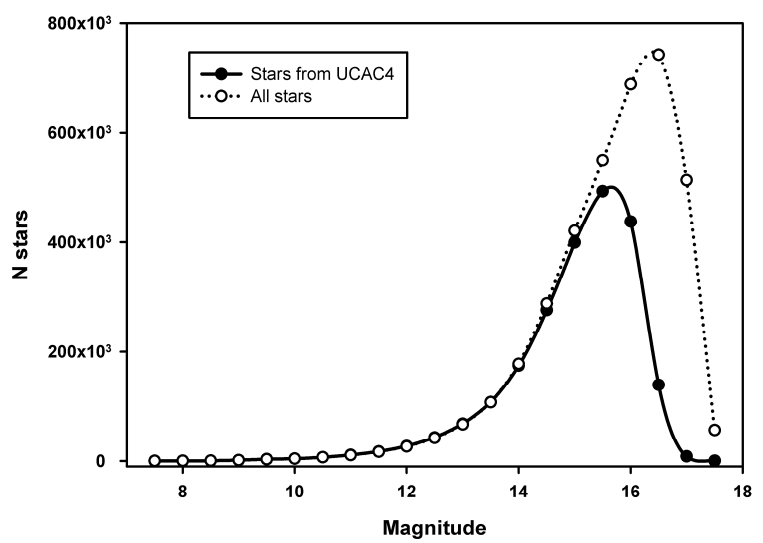

Figure 17: Distribution of stars of MOBITEL-2014 set catalog to magnitude

As clearly shown in Figure 19 the accuracy distribution to declination have the dependence from the zenith distance. Perhaps the reason is related to the lack of telescope alignment in south, which is usually performed only in the direction of the zenith $\left(\delta=47^{\circ}\right)$ and pole $\left(\delta=90^{\circ}\right)$ without alignment control to south of the zenith.

\section{Conclusion}

By using software, created in NAO in 2014, at 500 selected areas of sky within a 7 days of continuous operation more than 145 thousand astronomical images obtained at various telescopes from 1952 to 1999 was found and downloaded. For processing the arrays of images, including 20 thousand of own observations, the complex of 10 virtual machines was created on 2 PCs. Complex was set up to automatic processing of large volumes of observations in parallel and worked about 14 full days. As a result, we received more than 235 million coordinates of sky objects with good precision. All the processing is performed in a single system with the same software. And we obtained astrometric catalogs with more than 38 million stars.

Thus, the implementation of this investigation resulted in the increase performance of processes from 10 to 200 times. It shows how important for modern astronomy is to use modern information technology when working with large volumes of information.

Acknowledgements. The authors are thankful to Herbert Raab for the Astrometrica program, Yevgen Kozyryev for the Astrometrica automation program and anybody who has read this contribution to the end.

\section{References}

Mazhaev A. et al.: 2014, Odessa Astron. Publ., 27/1, 55.

Protsyuk Yu.I. et al.: 2007, Proc. of IAU Symp., N248, 548.

Protsyuk Yu.I. et al.: 2014, Kinem. Phys. Cel. Bodies, 30, N6, 54.

Skrutskie M.F. et al. : 2006, $A J, \mathbf{1 3 1}, 1163$.

Vavilova I. B. et al.: 2010, Kosmichna Nauka i Tekhn., 16, N5, 62 (in russian).

Vavilova I. B. et al.: 2011, Kosmichna Nauka i Tekhn., 17, N4, 74 (in russian).

Vavilova I.B. et al.: 2012, Kinem. Phys. Cel. Bodies, 28, N2, 85 .

Vavilova I. et al.: 2014, Odessa Astron. Publ., 27/1, 65. VizieR Online Data Catalog: 2005, The DENIS database Zacharias N. et al.: 2013, AJ, 145, 44 